Supporting information for:

\title{
A New Route to Annulated Oligothiophenes
}

Valentine G. Nenajdenko,* Viktor V. Sumerin, Konstantin Yu. Chernichenko and Elizabeth S. Balenkova

Moscow State University, Department of Chemistry, Leninskie Gory, Moscow 119992, Russia nen@acylium.chem.msu.ru

General Experimental. ${ }^{1} \mathrm{H}$ NMR and ${ }^{13} \mathrm{C}$ spectra were obtained using on Varian VXR-400 spectrometer in $\mathrm{CDCl}_{3}$ with TMS as an internal standard. Chemical shifts are reported in ppm. GCMS analyses were performed with an Finnigan SSQ 7000 Mass Spectrometer connected to a Varian 3400 GC, equipped with a DB-5MS column ( $30 \mathrm{~m}, 0.2 \mathrm{~mm}$ of internal diameter), electron impact (EI) at $70 \mathrm{eV}$ and $\mathrm{He}$ was used as the carrier gas. Merck $60 \mathrm{~F}_{254}$ plates were used for analytical (TLC) chromatography. Column chromatography was performed on silica gel (63-200 mesh, Merck). All new compounds gave satisfactory $400 \mathrm{MHz}{ }^{1} \mathrm{H}$ - and $100 \mathrm{MHz}{ }^{13} \mathrm{C}-\mathrm{NMR}$ spectral data. Melting points are uncorrected. All starting materials are commercially available.

3-\{[(Thien-3-ylthio)methyl]thio\}thiophene (3). To a solution of 3-bromothiophene (81.5 g, 46.8 $\mathrm{mL}, 0.50 \mathrm{~mol})$ in ether $(800 \mathrm{~mL}) t$-butyllithium $(687.0 \mathrm{~mL}, 1.03 \mathrm{~mol}, 1.5 \mathrm{M}$ in $n$-pentanes) was added dropwise at $-80^{\circ} \mathrm{C}$. The resulting mixture was stirred at this temperature for $15 \mathrm{~min}$. After warming to $-40^{\circ} \mathrm{C}$, sulfur powder $(15.8 \mathrm{~g}, 0.493 \mathrm{~mol})$ was added in one portion keeping the temperature below $-30^{\circ} \mathrm{C}$. The cooling bath was removed and the mixture was allowed to warm to r.t. for $1.5 \mathrm{~h}$. The solvent was removed in vacuo and the residue was dissolved in DMF (500 mL). At this solution dibromomethane ( $42.6 \mathrm{~g}, 17.2 \mathrm{~mL}, 0.245 \mathrm{~mol}$ ) was added dropwise. After heating for 4 $\mathrm{h}$ at $60^{\circ} \mathrm{C}$ the mixture was passed through a short column (silica gel; DMF). The solvent was removed by rotary evaporation, and the residue was dissolved in $\mathrm{CH}_{2} \mathrm{Cl}_{2}(500 \mathrm{~mL})$, washed with water (200 mL), dried over $\mathrm{K}_{2} \mathrm{CO}_{3}$ and finally evaporated. After distillation in portion (not more 0.05 mol, b.p. $137^{\circ} / 10^{-1}$ Torr) afforded $50.9 \mathrm{~g}$ of $\mathbf{3}$ (yield 85\%) as an yellow oil, $\mathrm{R}_{\mathrm{f}}(\mathrm{Hexane} / \mathrm{AcOEt}=$ 4:1) = 0.63. ${ }^{1} \mathrm{H}$ NMR (400 MHz, $\mathrm{CDCl}_{3}$ ): $\delta=7.33-7.31$ (m, 4H), 7.04 (t, $\left.J=3.2 \mathrm{~Hz}, 2 \mathrm{H}\right), 4.15$ (s, 2H). ${ }^{13} \mathrm{C}$ NMR (100 MHz, $\left.\mathrm{CDCl}_{3}\right): \delta=130.33,130.12,126.39,126.20$, 43.18. MS (EI, $\left.70 \mathrm{eV}\right) ; \mathrm{m} / \mathrm{z}$ (\%): 244 (29) $\left[\mathrm{M}^{+}\right], 129$ (100) $\left[\mathrm{M}^{+}-\mathrm{C}_{4} \mathrm{H}_{3} \mathrm{~S}_{2}\right]$. Anal. Calcd for $\mathrm{C}_{9} \mathrm{H}_{8} \mathrm{~S}_{4}$ : C, 44.22; H, 3.30. Found: C, 43.96; H, 3.35 . 
3-Bromo-2-(\{[(3-bromothienyl-2-yl)thio]methyl\}thio)thiophene (4a). To a solution of diisopropylamine $(60.7 \mathrm{~g}, 84.1 \mathrm{~mL}, 0.600 \mathrm{~mol})$ in ether $(800 \mathrm{~mL}) \mathrm{n}$-butyllithium $(206.0 \mathrm{~mL}, 0.515$ mol, $2.5 \mathrm{M}$ in $n$-hexanes) was added dropwise at $-30^{\circ} \mathrm{C}$. The mixture was allowed to warm to $0^{\circ} \mathrm{C}$ for $15 \mathrm{~min}$ and recooled to $-30^{\circ} \mathrm{C}$. To the above solution 3-bromothiophene $(81.5 \mathrm{~g}, 46.8 \mathrm{~mL}, 0.500$ mol) was added in one portion. The mixture was allowed to warm to $0^{\circ} \mathrm{C}$ for $1.5 \mathrm{~h}$ and was stirred at this temperature for $3 \mathrm{~h}$. The solution was recooled to $-40^{\circ} \mathrm{C}$ and sulfur powder $(15.8 \mathrm{~g}, 0.493 \mathrm{~mol})$ was added in one portion keeping the temperature below $-30^{\circ} \mathrm{C}$. After this addition, the cooling bath was removed and the mixture was allowed to warm to r.t. for $1.5 \mathrm{~h}$. The solvent was removed in vacuo and the residue was dissolved in DMF (500 mL). At this solution dibromomethane $(85.2 \mathrm{~g}$, $34.4 \mathrm{~mL}, 0.490 \mathrm{~mol}$ ) was added dropwise. After heating for $3 \mathrm{~h}$ at $100^{\circ} \mathrm{C}$ the mixture was allowed to cool to $80^{\circ} \mathrm{C}$ and poured into water $(1000 \mathrm{~mL})$. The precipitated product was filtered off, washed with water $(2 * 200 \mathrm{~mL})$, dissolved in toluene $(500 \mathrm{~mL})$, dried over $\mathrm{K}_{2} \mathrm{CO}_{3}$ and passed through a short column (silica gel; toluene). The solvent was removed by rotary evaporation, and the residue was purified by flash chromatography (silica gel; hot $\left(60^{\circ} \mathrm{C}\right) n$-hexane/toluene, 2:1). The crude product (84 g) was dissolved in mixture ether/hexane $(1: 2,600 \mathrm{~mL})$; the resulting solution was concentrated to $200 \mathrm{~mL}$ volume. White crystals precipitated and were filtered off after cooling to $0^{\circ} \mathrm{C}$, washed with hexane and dried to give $63.5 \mathrm{~g}$ (yield 65\%) of $\mathbf{4 a}, \mathrm{R}_{\mathrm{f}}($ Hexane/AcOEt $=4: 1)=$ 0.43. Mp 74.5-76 ${ }^{\circ}$ C. ${ }^{1} \mathrm{H}$ NMR (400 MHz, $\left.\mathrm{CDCl}_{3}\right): \delta=7.38$ (d, $\left.J=5.6 \mathrm{~Hz}, 2 \mathrm{H}\right), 7.04$ (d, $J=5.6 \mathrm{~Hz}$, 2H), 4.15 (s, 2H). ${ }^{13} \mathrm{C}$ NMR (100 MHz, $\left.\mathrm{CDCl}_{3}\right): \delta=130.94,130.15,128.21,119.48$, 45.18. MS (EI,

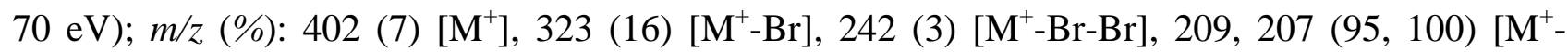
$\mathrm{C}_{4} \mathrm{H}_{2} \mathrm{BrS}_{2}$ ], 128 (42) [ $\left.\mathrm{M}^{+}-\mathrm{C}_{4} \mathrm{H}_{2} \mathrm{BrS}_{2}-\mathrm{Br}\right]$. Anal. Calcd for $\mathrm{C}_{9} \mathrm{H}_{6} \mathrm{Br}_{2} \mathrm{~S}_{4}$ : C, 26.88; H, 1.50. Found: C, 26.59; H, 1.54 .

2-Bromo-3-(\{[(2-bromothien-3-yl)thio]methyl\}thio)thiophene (4b). To a stirred solution of 3 (74.0 g, $0.303 \mathrm{~mol})$ in $\mathrm{CHCl}_{3}(1000 \mathrm{~mL})$ was added NBS (107.8 g, $\left.0.606 \mathrm{~mol}\right)$ over $30 \mathrm{~min}$. The mixture was stirred for $24 \mathrm{~h}$ at r.t., then $\mathrm{H}_{2} \mathrm{O}(1000 \mathrm{~mL})$ was added and the product was extracted with $\mathrm{CH}_{2} \mathrm{Cl}_{2}(100 \mathrm{~mL})$. The organic layer was washed with water (3*150 mL), aq $\mathrm{Na}_{2} \mathrm{CO}_{3}$ and dried over $\mathrm{K}_{2} \mathrm{CO}_{3}$. The solvent was removed by rotary evaporation, and the crude product was purified by column chromatography (silica gel; $n$-hexane/AcOEt, 50:1 ) to afford 109.6 g of $\mathbf{4 b}$ (yield 90\%) as a yellow oil (unstable and should be used immediately in next step), $\mathrm{R}_{\mathrm{f}}($ Hexane/AcOEt $=4: 1)=0.43$. ${ }^{1} \mathrm{H}$ NMR (400 MHz, $\mathrm{CDCl}_{3}$ ): $\delta=7.25$ (d, $\left.J=5.9 \mathrm{~Hz}, 2 \mathrm{H}\right), 7.00$ (d, $\left.J=5.9 \mathrm{~Hz}, 2 \mathrm{H}\right), 4.20(\mathrm{~s}, 2 \mathrm{H}) .{ }^{13} \mathrm{C}$ NMR (100 MHz, $\left.\mathrm{CDCl}_{3}\right): \delta=131.00,126.04,116.58,107.59,41.24$. MS (EI, $\left.70 \mathrm{eV}\right) ; \mathrm{m} / \mathrm{z}(\%): 402$ 


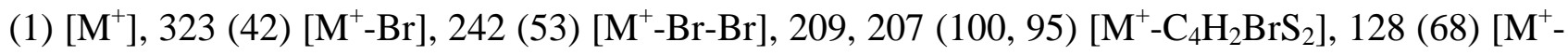
$\left.\mathrm{C}_{4} \mathrm{H}_{2} \mathrm{BrS}_{2}-\mathrm{Br}\right]$. Anal. Calcd for $\mathrm{C}_{9} \mathrm{H}_{6} \mathrm{Br}_{2} \mathrm{~S}_{4}$ : C, 26.88; H, 1.50. Found: C, 26.85; H, 1.55.

10H-Bisthieno[2,3-d:3',2'-g][1,3]dithiocin-10-one (5a). Compound 4a (63.3 g, 0.157 mol) was dissolved in dry ether $\left(4000 \mathrm{~mL}\right.$ ) and cooled to $-78^{\circ} \mathrm{C}$; then $t$ - $\mathrm{BuLi}(420 \mathrm{~mL}, 0.630 \mathrm{~mol}, 1.5 \mathrm{M}$ in $n$ pentane) was added dropwise. The resulting mixture was stirred for $1 \mathrm{~h}$ during which time, the temperature was allowed to rise to $-40^{\circ} \mathrm{C}$. The yellow solution thus obtained was cooled to $-50^{\circ} \mathrm{C}$ and ethyl $N, N^{\prime}$-dimethylcarbamate (64.5 g, $0.551 \mathrm{~mol}$ ) was added dropwise over 1 to $2 \mathrm{~min}$. The mixture was allowed to warm to $-40^{\circ} \mathrm{C}$, then stirred for $4 \mathrm{~h}$ at temperatures between -40 to $-30^{\circ} \mathrm{C}$. After standing overnight at r.t., the solvent was evaporated in vacuo and the residue was dissolved in hot $\left(60^{\circ} \mathrm{C}\right) \mathrm{CHCl}_{3}(1000 \mathrm{~mL})$, washed with water $(200 \mathrm{~mL})$, dried over $\mathrm{K}_{2} \mathrm{CO}_{3}$ and passed through a short column (silica gel; $\mathrm{CHCl}_{3}$ ). The solvent was removed by rotary evaporation, and the crude product (34 g) was purified by column chromatography (silica gel; eluted with a 2-10\% AcOEt in $n$ hexane gradient) to afford $27.65 \mathrm{~g}$ of $\mathbf{5 a}$ (yield $65 \%$ ) as an orange oil. After standing for a long time at r.t., the resulting oil solidified to give orange crystals, $R_{f}($ Hexane/AcOEt $=4: 1)=0.40$. Mp 63.064. $0^{\circ} \mathrm{C} .{ }^{1} \mathrm{H}$ NMR $\left(400 \mathrm{MHz}, \mathrm{CDCl}_{3}\right): \delta=7.60(\mathrm{~d}, J=5.6 \mathrm{~Hz}, 2 \mathrm{H}), 7.43(\mathrm{~d}, J=5.6 \mathrm{~Hz}, 2 \mathrm{H}), 3.83(\mathrm{~s}$, 2H). ${ }^{13} \mathrm{C}$ NMR $\left(\mathrm{CDCl}_{3}\right): \delta=183.01,148.63,132.41,130.77,130.03,48.93 . \mathrm{MS}(\mathrm{EI}, 70 \mathrm{eV}) ; \mathrm{m} / \mathrm{z}$ (\%): $270(100)\left[\mathrm{M}^{+}\right], 224(68)\left[\mathrm{M}^{+}-\mathrm{CH}_{2} \mathrm{~S}\right], 142(71)\left[\mathrm{M}^{+}-\mathrm{C}_{5} \mathrm{H}_{4} \mathrm{~S}_{2}\right]$. Anal. Calcd for $\mathrm{C}_{10} \mathrm{H}_{6} \mathrm{OS}_{4}$ : C, 44.42; H, 2.24. Found: C, 44.49; H, 2.42.

10H-Bisthieno[2,3-g:3',2'-d][1,3]dithiocin-10-one (5b). Compound 4b (31.7 g, 0.079 mol) was dissolved in dry ether $(2000 \mathrm{~mL})$ and cooled to $-78^{\circ} \mathrm{C}$; then $t-\mathrm{BuLi}(210 \mathrm{~mL}, 0.315 \mathrm{~mol}, 1.5 \mathrm{M}$ in $n$ pentane) was added dropwise. The resulting mixture was stirred for $1 \mathrm{~h}$ during which time, the temperature was allowed to rise to $-40^{\circ} \mathrm{C}$. The yellow solution thus obtained was cooled to $-50^{\circ} \mathrm{C}$ and ethyl $N, N^{\prime}$-dimethylcarbamate ( $32.3 \mathrm{~g}, 0.275 \mathrm{~mol}$ ) was added dropwise over 1 to $2 \mathrm{~min}$. The mixture was allowed to warm to $-40^{\circ} \mathrm{C}$, then stirred for $4 \mathrm{~h}$ at temperatures between -40 to $-30^{\circ} \mathrm{C}$. After standing overnight at r.t., the solvent was evaporated in vacuo and the residue was dissolved in hot $\left(60^{\circ} \mathrm{C}\right) \mathrm{CHCl}_{3}(1000 \mathrm{~mL})$, washed with water $(200 \mathrm{~mL})$, dried over $\mathrm{K}_{2} \mathrm{CO}_{3}$ and passed through a 
short column (silica gel; $\mathrm{CHCl}_{3}$ ). The solvent was removed by rotary evaporation, and the crude product (20 g) was purified by column chromatography (silica gel; eluted with a 2-10\% AcOEt in $n$ hexane gradient) to afford $13.8 \mathrm{~g}$ of $\mathbf{5 b}$ (yield $65 \%$ ) as an yellow crystals, $\mathrm{R}_{\mathrm{f}}($ Hexane/AcOEt $=4: 1)$ $=0.40 . \mathrm{Mp} 131.5^{\circ}-133.0^{\circ} \mathrm{C} .{ }^{1} \mathrm{H} \operatorname{NMR}\left(400 \mathrm{MHz}, \mathrm{CDCl}_{3}\right): \delta=7.70(\mathrm{~d}, J=5.0 \mathrm{~Hz}, 2 \mathrm{H}), 7.13(\mathrm{~d}, J=$ $5.0 \mathrm{~Hz}, 2 \mathrm{H}), 3.91$ (s, 2H). ${ }^{13} \mathrm{C}$ NMR (100 MHz, $\left.\mathrm{CDCl}_{3}\right): \delta=180.19,149.94,135.37,132.66,130.00$,

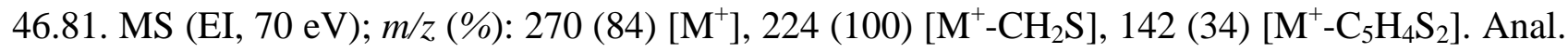
Calcd for $\mathrm{C}_{10} \mathrm{H}_{6} \mathrm{OS}_{4}$ : C, 44.42; H, 2.24. Found: C, 44.52; H, 2.36.

Intramolecular Coupling of 5a and 5b. Compound 5a (2.705 g, $10.0 \mathrm{mmol})$ was dissolved in mixture ether/HMPA $(9: 1,1000 \mathrm{~mL})$ and cooled to $-78^{\circ} \mathrm{C}$; $t$ - BuLi $(6.67 \mathrm{~mL}, 10.0 \mathrm{mmol}, 1.5 \mathrm{M}$ in $n$ pentane,) was then added dropwise. The resulting mixture was stirred for $2 \mathrm{~h}$ at this temperature, then at r.t. overnight and quenched by addition of water $(50 \mathrm{~mL})$. The ether was removed by rotary evaporation $\left(20^{\circ} \mathrm{C}\right)$ and the residue was heating for $1 \mathrm{~h}$ at $150-200^{\circ} \mathrm{C}$. The HMPA was removed in vacuo $\left(100^{\circ} \mathrm{C} / 7\right.$ torr) then water was added and the product was extracted twice with $\mathrm{CHCl}_{3}(50$ $\mathrm{mL})$. The combined organic layers were washed with water $(3 * 25 \mathrm{~mL})$, dried over $\mathrm{K}_{2} \mathrm{CO}_{3}$ and concentrated in vacuo. The crude product (1.50 g) was passed through a short column (silica gel; hexane $/ \mathrm{CHCl}_{3}, 2: 1$ ) to afford $1.36 \mathrm{~g}$ of 1a (yield 54\%) after evaporation of solvent and crystallization from hexane/ $\mathrm{CHCl}_{3}$ (2:1). A similar reaction of $5 \mathbf{b}$ produced $\mathbf{5 a}$ in $50 \%$ yield.

Thieno[2,3-b]thieno[3',2':4,5]thieno[3,2-d]thiophene (1a). $\mathrm{R}_{\mathrm{f}}($ Hexane/AcOEt $=4: 1)=0.51$. Mp 159.5-161.0 ${ }^{\circ} \mathrm{C} .{ }^{1} \mathrm{H}$ NMR (400 MHz, DMSO- $\left.d^{6}\right): \delta=7.79$ (B part of an AB system, $J=5.1 \mathrm{~Hz}$, 2H), 7.71 (A part of an AB system, $J=5.1 \mathrm{~Hz}, 2 \mathrm{H}) .{ }^{13} \mathrm{C}$ NMR (100 MHz, DMSO- $d^{6}$ ): $\delta=140.91$, 138.54, 137.26, 129.15, 118.90, 115.32. MS (EI, $70 \mathrm{eV}) ; \mathrm{m} / \mathrm{z}(\%): 252$ (100) $\left[\mathrm{M}^{+}\right]$. Anal. Calcd for $\mathrm{C}_{10} \mathrm{H}_{4} \mathrm{~S}_{4}$ : C, 47.59; H, 1.60. Found: C, 47.68; H, 1.75.

Thieno[3,2-b]thieno[2',3':4,5]thieno[3,2-d]thiophene (1b). $\mathrm{R}_{\mathrm{f}}($ Hexane/AcOEt $=4: 1)=0.51$. Mp 169.5-171.0 ${ }^{\circ}$ C. ${ }^{1} \mathrm{H}$ NMR (400 MHz, DMSO- $\left.d^{6}\right): \delta=7.76$ (d, $\left.J=5.1 \mathrm{~Hz}, 2 \mathrm{H}\right), 7.56(\mathrm{~d}, J=5.1$ Hz, 2H). ${ }^{13} \mathrm{C}$ NMR (100 MHz, DMSO- $\left.d^{6}\right): \delta=141.70,141.10,128.31,127.21,121.45,115.32$. MS (EI, $70 \mathrm{eV}$ ); m/z (\%): 252 (100) $\left[\mathrm{M}^{+}\right]$. Anal. Calcd for $\mathrm{C}_{10} \mathrm{H}_{4} \mathrm{~S}_{4}$ : C, 47.59; H, 1.60. Found: C, 47.65; H, 1.91 . 


\section{X-Ray structure of compound 1a:}

Table 1. Crystal data and structure refinement for bal02.

Identification code

Empirical formula

Formula weight

Temperature

Wavelength

Crystal system, space group

Unit cell dimensions

Volume

Z, Calculated density

Diffractometer

Collection method

Radiation type

Radiation

Standards number

Standards interval time

Standards interval count

Standards decay bal02

C10 H4 S4

252.37

293(2) K

$0.71073 \mathrm{~A}$

Orthorombic, C222(1)

$\mathrm{a}=4.776(1)$ A alpha $=90$ deg.

$\mathrm{b}=13.918(3)$ A beta $=90$ deg.

$\mathrm{c}=14.700(3)$ A gamma $=90 \mathrm{deg}$.

977.1(4) $\mathrm{A} \wedge 3$

4, $1.716 \mathrm{Mg} / \mathrm{m}^{\wedge} 3$

CAD4

theta $\backslash 2$ theta

MoKla

monochromator filter

3

$100 \mathrm{~min}$

?

$0.3 \%$ 
Theta range for data collection

Limiting indices

Reflections collected / unique

Completeness to theta $=25.97$

Absorption correction

Absorption coefficient

$\mathrm{F}(000)$

Crystal description

Crystal colour

Crystal size

Max. and min. transmission

Refinement method

Data / restraints / parameters

Goodness-of-fit on

Final R indices [I>2sigma(I)]

$\mathrm{R}$ indices (all data)

Absolute structure parameter

Extinction coefficient

Largest diff. peak and hole
2.77 to 25.97 deg.

$-5<=\mathrm{h}<=0,-16<=\mathrm{k}<=0,-18<=\mathrm{l}<=18$

$1105 / 958[\mathrm{R}(\mathrm{int})=0.0356]$

$100.0 \%$

None

$0.919 \mathrm{~mm}^{\wedge}-1$

512

plate

colorless

$0.41 \times 0.38 \times 0.02 \mathrm{~mm}$

0.9818 and 0.7044

Full-matrix least-squares on $\mathrm{F}^{\wedge} 2$

958 / 0 / 74

$F^{\wedge} 21.012$

$\mathrm{R} 1=0.0270, \mathrm{wR} 2=0.0671$

$\mathrm{R} 1=0.0350, \mathrm{wR} 2=0.0686$

$-0.17(13)$

0.0019(17)

0.292 and -0.268 e. $\mathrm{A}^{\wedge}-3$ 
Table 2. Atomic coordinates ( $\left.\mathrm{x} 10^{\wedge} 4\right)$ and equivalent isotropic displacement parameters $(\mathrm{A} \wedge 2 \mathrm{x}$ 10^3) for bal02.

$\mathrm{U}(\mathrm{eq})$ is defined as one third of the trace of the orthogonalized Uij tensor.

\begin{tabular}{|c|c|c|c|c|}
\hline & $\mathrm{x}$ & $\mathrm{y}$ & $\mathrm{z}$ & $\mathrm{U}(\mathrm{eq})$ \\
\hline$S(1)$ & $5409(1)$ & $3854(1)$ & $4477(1)$ & $45(1)$ \\
\hline$S(2)$ & 2084(2) & 5403(1) & 3335(1) & $49(1)$ \\
\hline $\mathrm{C}(1)$ & $2790(5)$ & $2605(2)$ & $3482(2)$ & $40(1)$ \\
\hline$C(2)$ & $1850(4)$ & $3523(2)$ & 3183(2) & $36(1)$ \\
\hline C(3) & 0 & 3896(2) & 2500 & $35(1)$ \\
\hline$C(4)$ & $4710(5)$ & 2683(2) & $4165(2)$ & $45(1)$ \\
\hline$C(5)$ & $3100(5)$ & $4261(2)$ & $3671(2)$ & $40(1)$ \\
\hline C(6) & 0 & $4888(2)$ & 2500 & $43(1)$ \\
\hline
\end{tabular}


Table 3. Bond lengths [A] and angles [deg] for bal02.

\begin{tabular}{|c|c|}
\hline$S(1)-C(5)$ & $1.715(2)$ \\
\hline$S(1)-C(4)$ & $1.727(3)$ \\
\hline$S(2)-C(5)$ & $1.733(2)$ \\
\hline$S(2)-C(6)$ & $1.7353(15)$ \\
\hline $\mathrm{C}(1)-\mathrm{C}(4)$ & $1.364(4)$ \\
\hline$C(1)-C(2)$ & 1.424(3) \\
\hline$C(2)-C(5)$ & 1.388(3) \\
\hline C(2)-C(3) & $1.435(3)$ \\
\hline $\mathrm{C}(3)-\mathrm{C}(6)$ & $1.381(5)$ \\
\hline $\mathrm{C}(3)-\mathrm{C}(2) \# 1$ & $1.435(3)$ \\
\hline $\mathrm{C}(6)-\mathrm{S}(2) \# 1$ & $1.7353(15)$ \\
\hline$C(5)-S(1)-C(4)$ & $90.24(12)$ \\
\hline$C(5)-S(2)-C(6)$ & 89.07(12) \\
\hline$C(4)-C(1)-C(2)$ & $111.6(2)$ \\
\hline $\mathrm{C}(5)-\mathrm{C}(2)-\mathrm{C}(1)$ & $111.6(2)$ \\
\hline $\mathrm{C}(5)-\mathrm{C}(2)-\mathrm{C}(3)$ & $111.0(2)$ \\
\hline$C(1)-C(2)-C(3)$ & $137.3(2)$ \\
\hline$C(6)-C(3)-C(2) \# 1$ & $111.22(15)$ \\
\hline
\end{tabular}




$\begin{array}{ll}\mathrm{C}(6)-\mathrm{C}(3)-\mathrm{C}(2) & 111.22(15) \\ \mathrm{C}(2) \# 1-\mathrm{C}(3)-\mathrm{C}(2) & 137.6(3) \\ \mathrm{C}(1)-\mathrm{C}(4)-\mathrm{S}(1) & 113.57(19) \\ \mathrm{C}(2)-\mathrm{C}(5)-\mathrm{S}(1) & 112.91(18) \\ \mathrm{C}(2)-\mathrm{C}(5)-\mathrm{S}(2) & 114.26(18) \\ \mathrm{S}(1)-\mathrm{C}(5)-\mathrm{S}(2) & 132.81(15) \\ \mathrm{C}(3)-\mathrm{C}(6)-\mathrm{S}(2) & 114.38(10) \\ \mathrm{C}(3)-\mathrm{C}(6)-\mathrm{S}(2) \# 1 & 114.38(10) \\ \mathrm{S}(2)-\mathrm{C}(6)-S(2) \# 1 & 131.2(2)\end{array}$

Symmetry transformations used to generate equivalent atoms:

$\# 1-\mathrm{x}, \mathrm{y},-\mathrm{z}+1 / 2$ 
Table 4. Anisotropic displacement parameters $\left(\mathrm{A}^{\wedge} 2 \mathrm{2} \times 10^{\wedge} 3\right)$ for bal02.

The anisotropic displacement factor exponent takes the form:

$-2 \mathrm{pi}^{\wedge} 2\left[\mathrm{~h}^{\wedge} 2 \mathrm{a}^{* \wedge} \mathrm{2} \mathrm{U} 11+\ldots+2 \mathrm{~h} \mathrm{k} \mathrm{a*} \mathrm{b}^{*} \mathrm{U} 12\right]$

\begin{tabular}{lllllll}
\hline & $\mathrm{U} 11$ & $\mathrm{U} 22$ & $\mathrm{U} 33$ & $\mathrm{U} 23$ & $\mathrm{U} 13$ & $\mathrm{U} 12$ \\
$\mathrm{~S}(1)$ & $43(1)$ & $48(1)$ & $43(1)$ & $-6(1)$ & $-3(1)$ & $2(1)$ \\
$\mathrm{S}(2)$ & $57(1)$ & $33(1)$ & $56(1)$ & $-5(1)$ & $-7(1)$ & $-2(1)$ \\
$\mathrm{C}(1)$ & $44(1)$ & $35(1)$ & $42(1)$ & $-2(1)$ & $4(1)$ & $3(1)$ \\
$\mathrm{C}(2)$ & $32(1)$ & $37(1)$ & $40(1)$ & $-2(1)$ & $5(1)$ & $1(1)$ \\
$\mathrm{C}(3)$ & $36(1)$ & $33(1)$ & $36(1)$ & 0 & $5(1)$ & 0 \\
$\mathrm{C}(4)$ & $48(1)$ & $43(1)$ & $43(1)$ & $-3(1)$ & $-1(1)$ & $6(1)$ \\
$\mathrm{C}(5)$ & $38(1)$ & $38(1)$ & $43(1)$ & $-1(1)$ & $1(1)$ & $0(1)$ \\
$\mathrm{C}(6)$ & $48(2)$ & $34(2)$ & $46(2)$ & 0 & $-1(1)$ & 0 \\
\hline
\end{tabular}


Table 5. Hydrogen coordinates ( x 10^4) and isotropic displacement parameters $\left(\mathrm{A}^{\wedge} 2 \mathrm{x} 10^{\wedge} 3\right.$ ) for bal02.

\begin{tabular}{lllll}
\hline & $\mathrm{x}$ & $\mathrm{y}$ & $\mathrm{U}$ & \\
& & & \\
\hline $\mathrm{H}(1)$ & $2120(60)$ & $2001(18)$ & $3212(18)$ & $41(7)$ \\
$\mathrm{H}(4)$ & $5740(60)$ & $2200(20)$ & $4520(20)$ & $56(8)$ \\
\hline
\end{tabular}



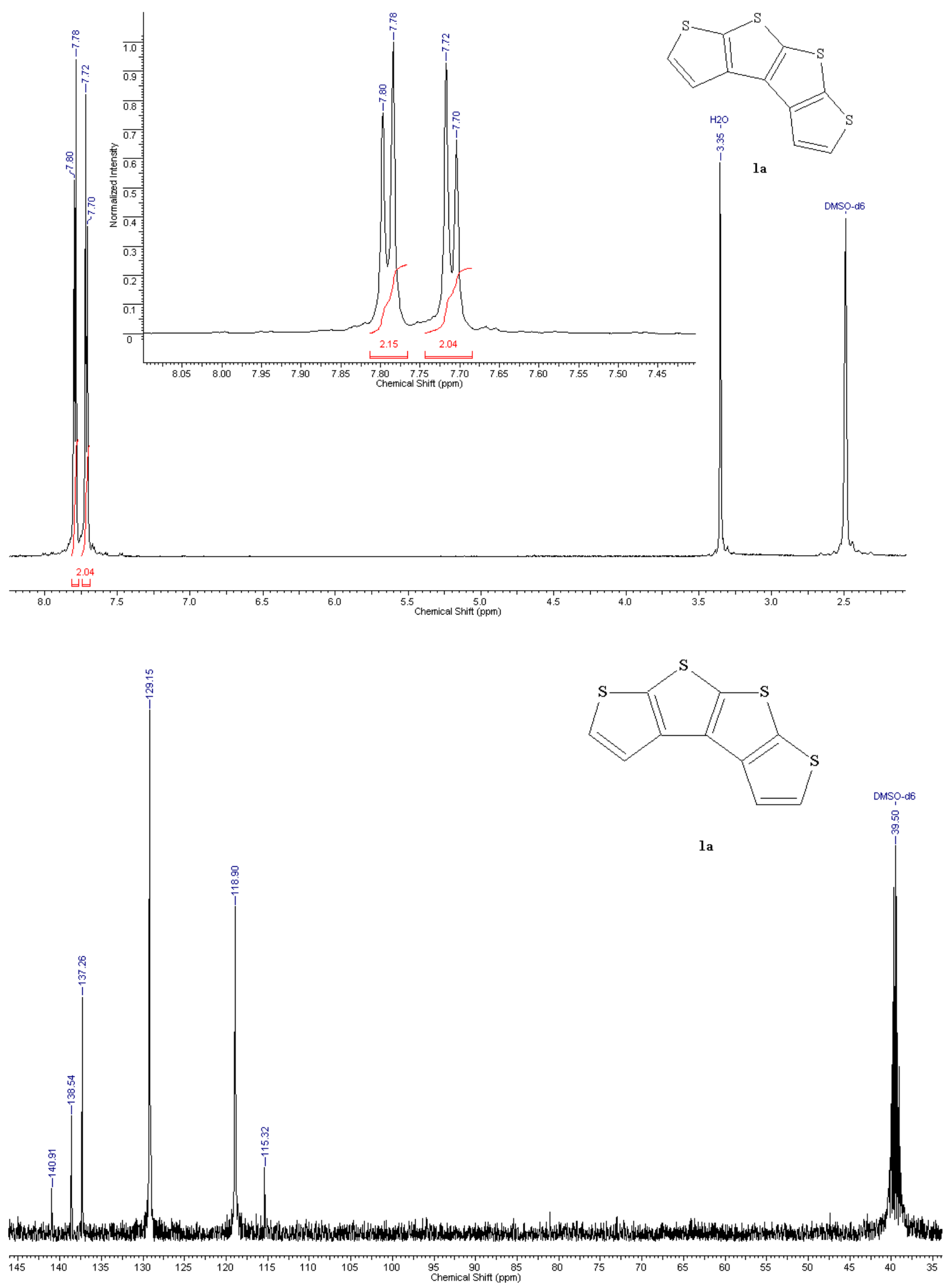


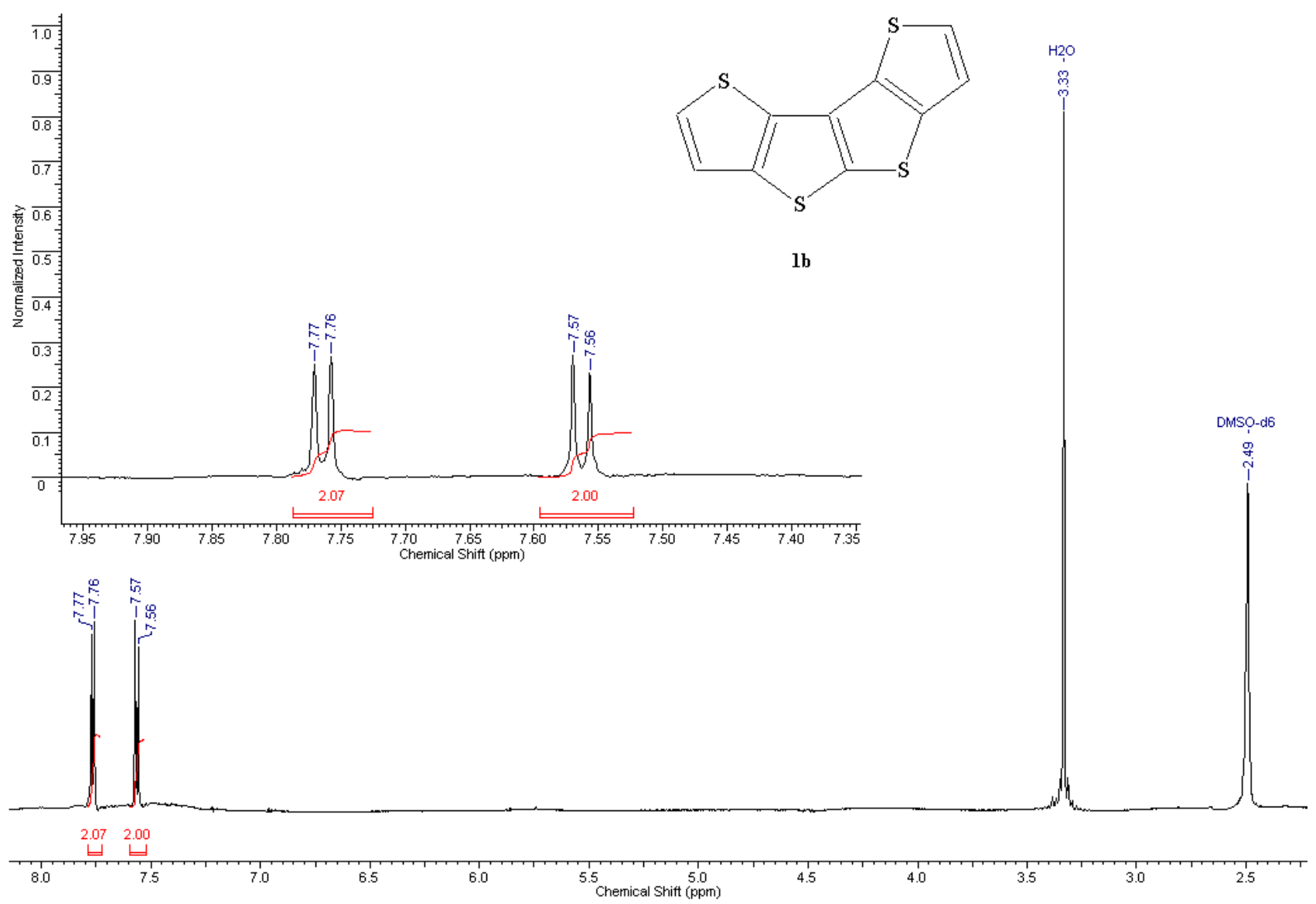




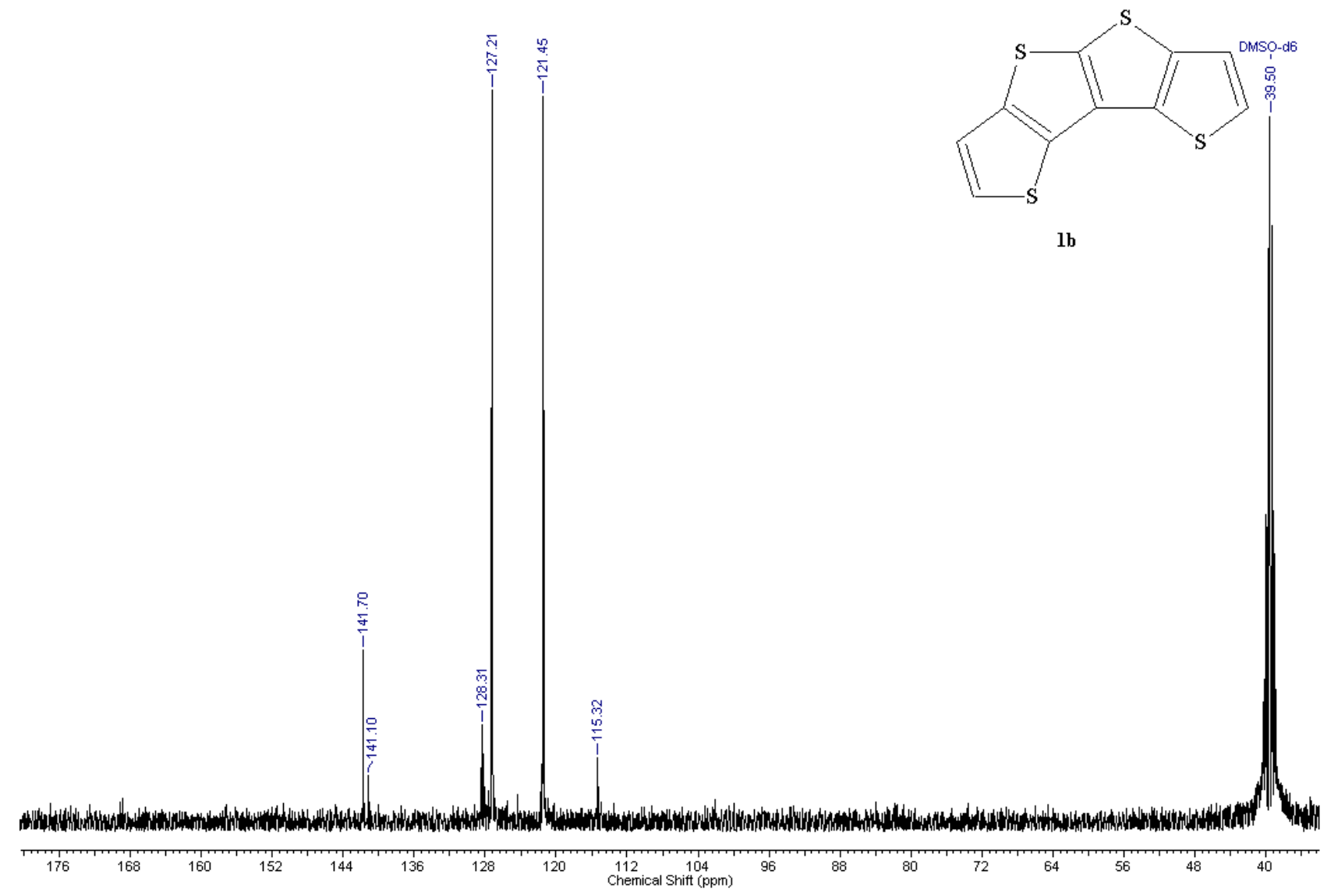



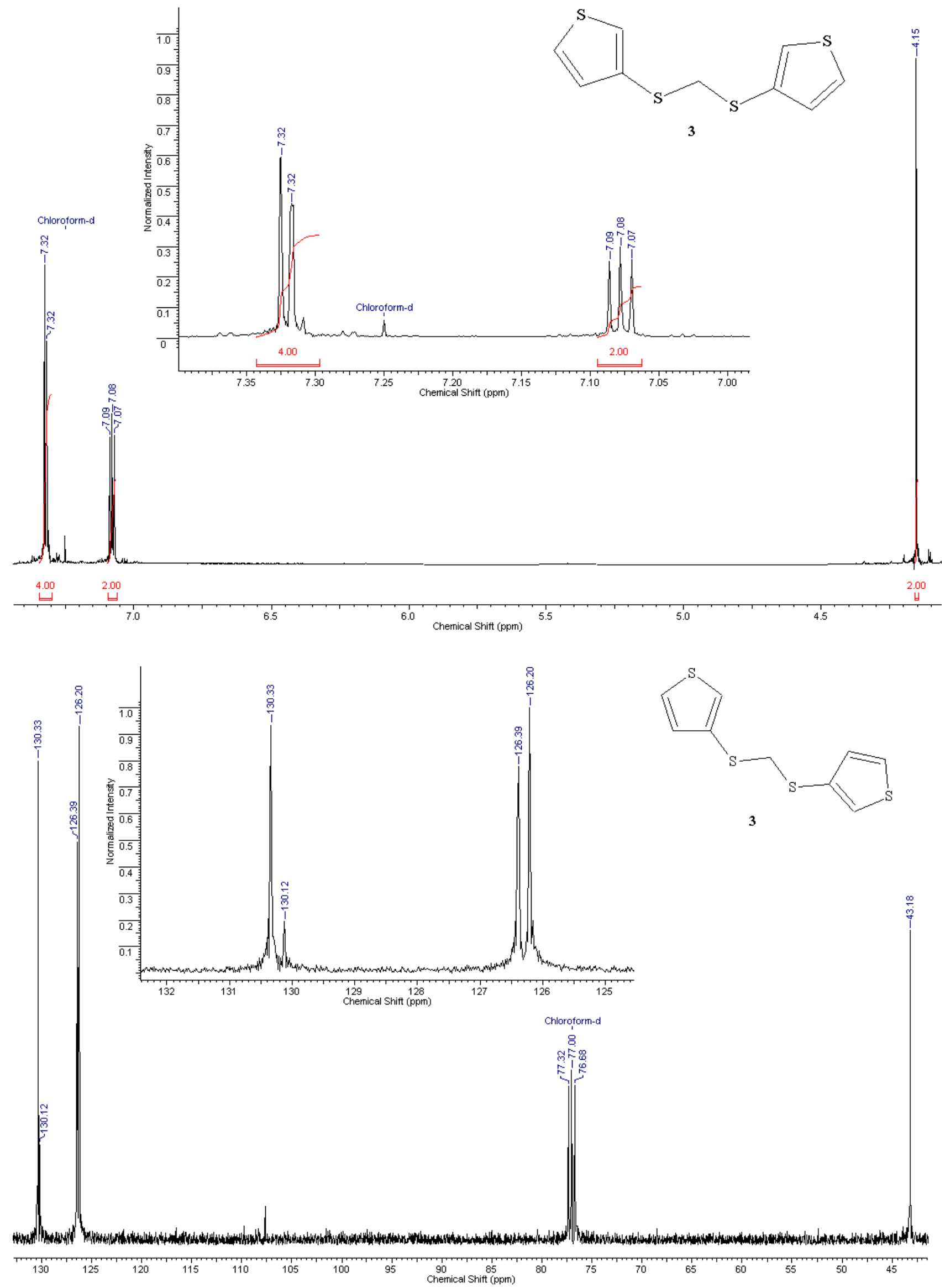

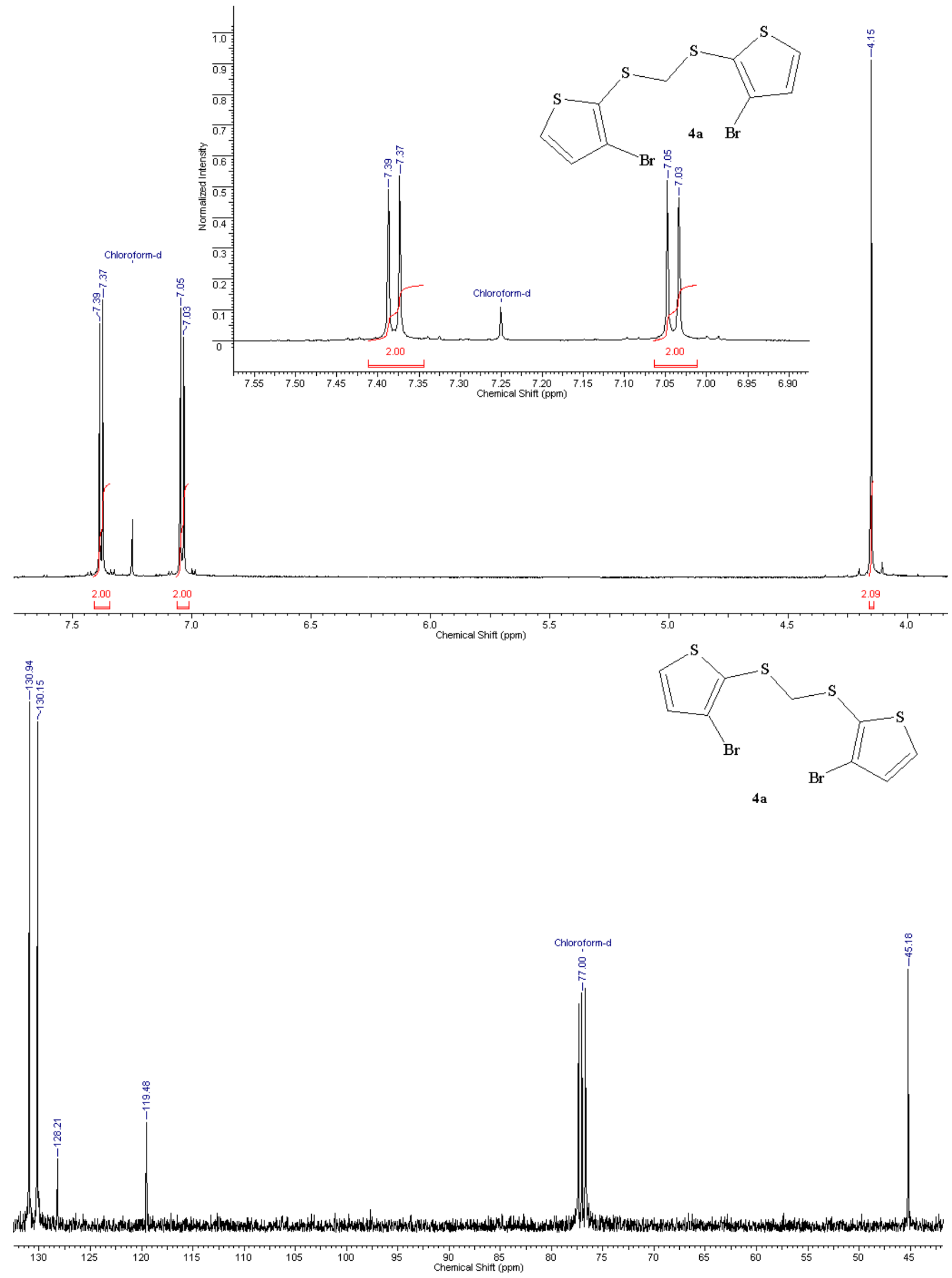

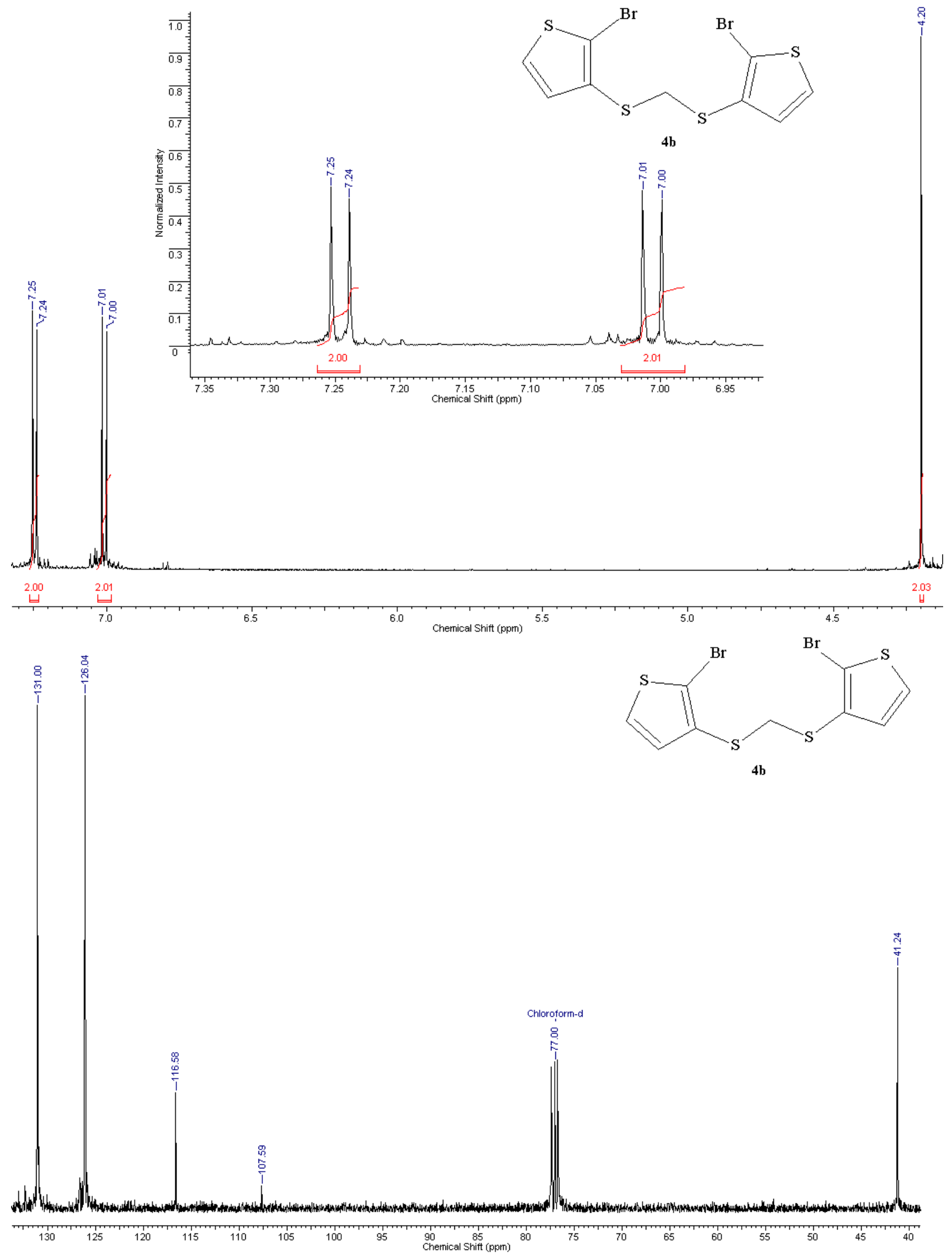


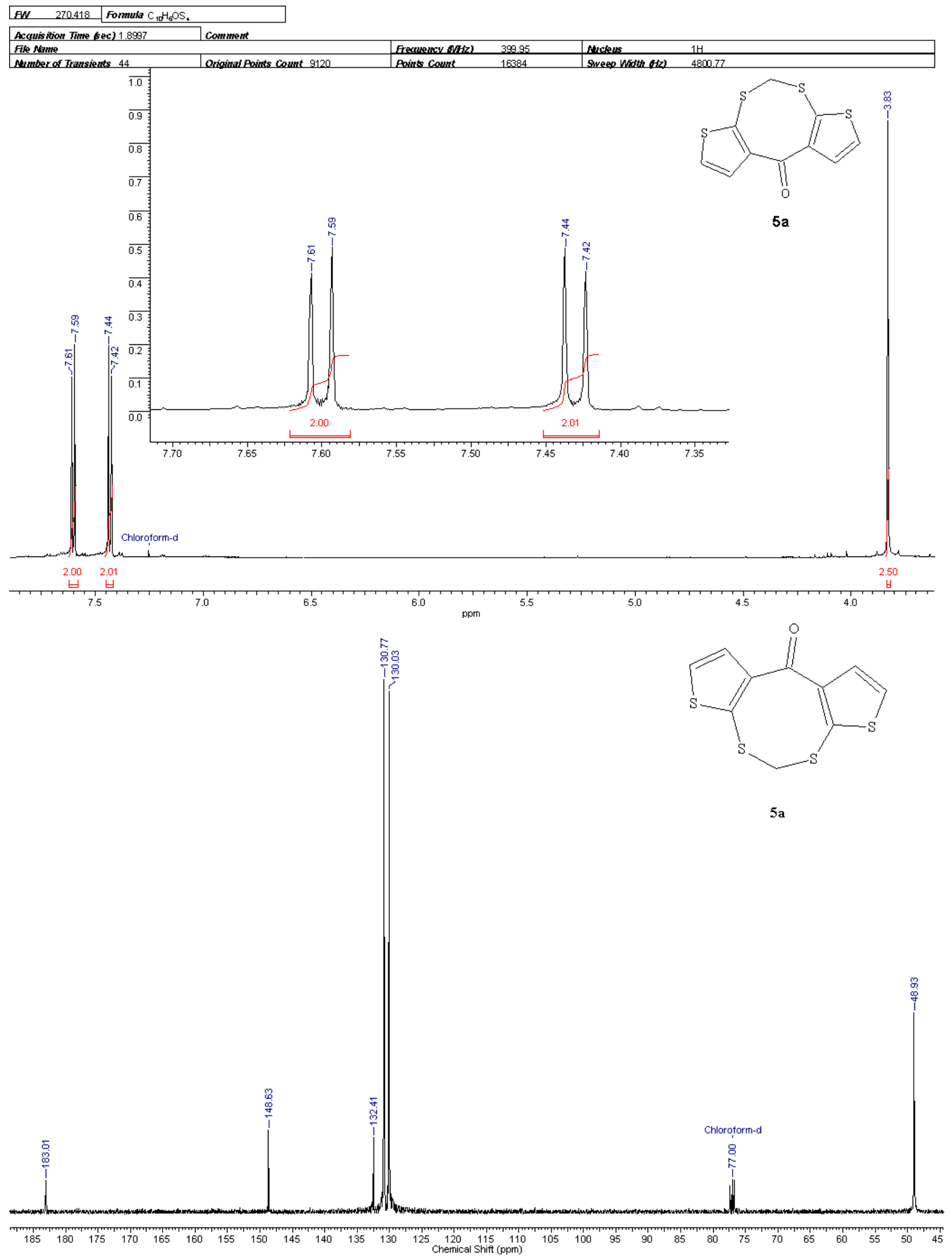




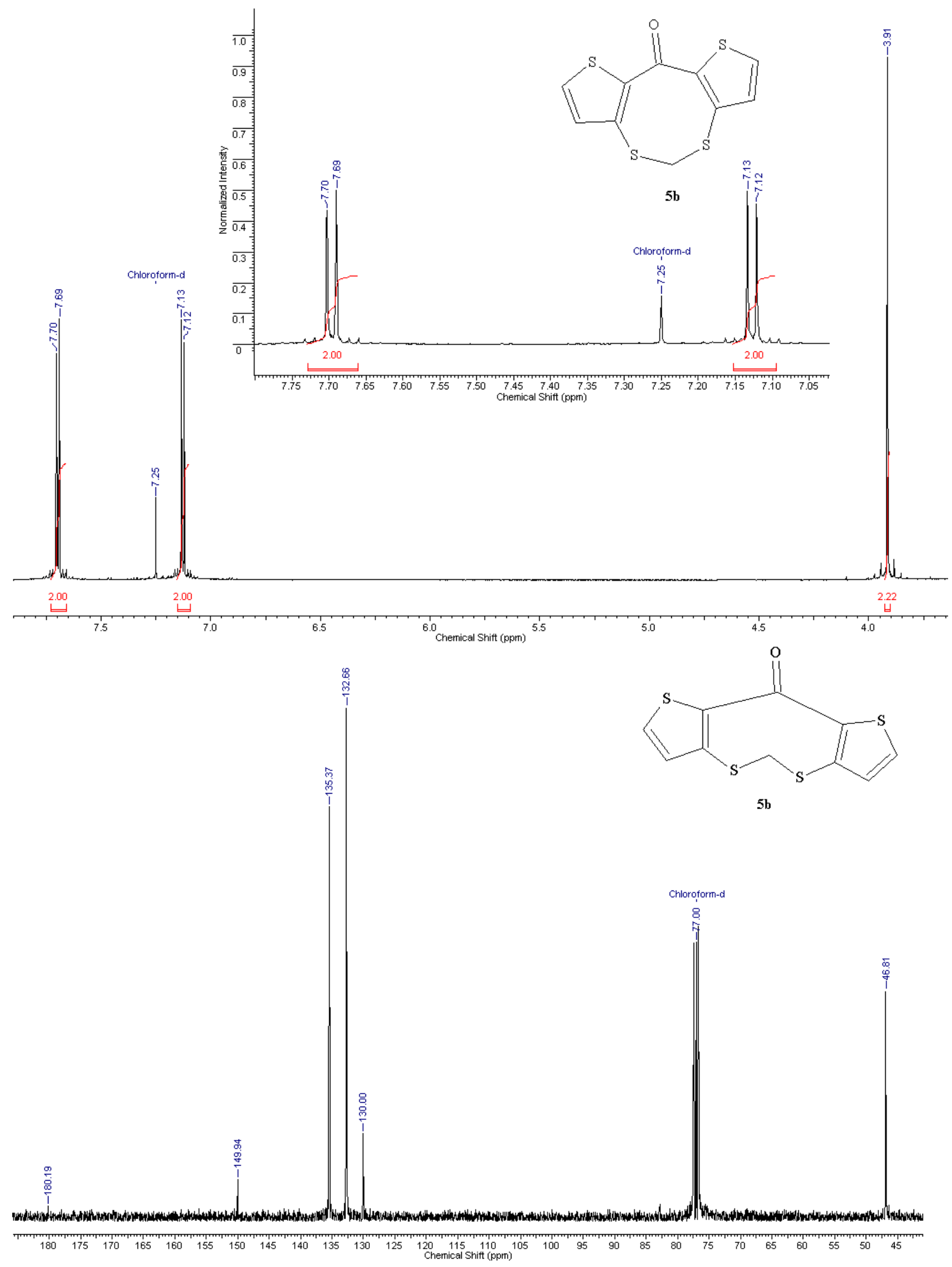

19 